Article

\title{
Forest Carbon Leakage Quantification Methods and Their Suitability for Assessing Leakage in REDD
}

\section{Sabine Henders ${ }^{1, *}$ and Madelene Ostwald ${ }^{1,2}$}

1 Center for Climate Science and Policy Research, Department of Water and Environmental Studies, Linköping University, Norrköping 601 74, Sweden

2 Physical Resource Theory, Department of Energy and Environment, Chalmers University of Technology, Göteborg 412 96, Sweden; E-Mail: madelene.ostwald@chalmers.se

* Author to whom correspondence should be addressed; E-Mail: sabine.henders@liu.se; Tel.: +46-11-363377; Fax: +46-11-363292.

Received: 31 October 2011; in revised form: 15 December 2011 / Accepted: 6 January 2012 / Published: 16 January 2012

\begin{abstract}
This paper assesses quantification methods for carbon leakage from forestry activities for their suitability in leakage accounting in a future Reducing Emissions from Deforestation and Forest Degradation (REDD) mechanism. To that end, we first conducted a literature review to identify specific pre-requisites for leakage assessment in REDD. We then analyzed a total of 34 quantification methods for leakage emissions from the Clean Development Mechanism (CDM), the Verified Carbon Standard (VCS), the Climate Action Reserve (CAR), the CarbonFix Standard (CFS), and from scientific literature sources. We screened these methods for the leakage aspects they address in terms of leakage type, tools used for quantification and the geographical scale covered. Results show that leakage methods can be grouped into nine main methodological approaches, six of which could fulfill the recommended REDD leakage requirements if approaches for primary and secondary leakage are combined. The majority of methods assessed, address either primary or secondary leakage; the former mostly on a local or regional and the latter on national scale. The VCS is found to be the only carbon accounting standard at present to fulfill all leakage quantification requisites in REDD. However, a lack of accounting methods was identified for international leakage, which was addressed by only two methods, both from scientific literature.
\end{abstract}


Keywords: forest carbon accounting standards; scientific literature; primary and secondary leakage; international leakage; national-scale accounting

\section{Introduction}

After its exclusion from the Kyoto Protocol in 2003, forest conservation in developing countries was brought up again as a potential and potent mitigation option at the climate talks during COP 11, 2005 in Montreal, in the form of a proposed mechanism for reducing emissions from deforestation and forest degradation (REDD) [1]. Since then, there has been an increased focus on the land use sector as solution to the climate change problem. At COP 13 in Bali the United Nations Framework Convention on Climate Change (UNFCCC) asked parties and international organizations to promote REDD through pilot projects and capacity building. This request has been followed by a frantic acceleration of work trying to understand and reduce the hurdles for such a mechanism [2]. Many of these hurdles are methodological, such as the establishment of baselines for deforestation emissions [3], which base year or base period to use [4], monitoring and verification systems and their requirements [3], how to guarantee long-term carbon storage for the future, also referred to as permanence [5], and the issue of carbon leakage [4], which is the subject of this paper.

Carbon leakage refers to the displacement of greenhouse gas (GHG) emissions from one place to another due to emission reduction activities. It is caused by a direct or indirect shift of activities that create those emissions from within an emissions accounting system to out of that system. The IPCC defines leakage as "the unanticipated decrease or increase in GHG benefits outside of the project's accounting boundary (...) as a result of the project activities” [6]. While this definition is mainly geared at local scale emission reduction project activities, leakage can also occur when emissions regulations or policies are adopted in one place, and emissions shift to elsewhere where this policy is not effective [7].

Although leakage can occur in any sector affected by GHG mitigation [8-10], it has historically mainly been discussed in connection with activities in the forestry sector, particularly for avoided deforestation or REDD. Contributing to the scientific and carbon-market debate of how to address leakage from forest conservation activities, in this paper we examine specific requirements that we consider essential for leakage accounting in REDD, and analyze existing leakage quantification methods to assess whether they match these requirements and are suitable to address leakage from REDD. While a national accounting scope for REDD as intended by the UNFCCC [11,12] would eventually lead to an internalization of leakage emissions into the accounting system, methods to identify and control leakage are expected to play an important role in REDD for two reasons: Firstly, even though a national-scale REDD inventory system implies that leakage emissions would be captured and accounted for, the underlying leakage processes as such are not prevented. Displacement of deforestation activities could still occur and undermine the overall efficiency of emission reduction activities; thus increasing the cost of REDD implementation [13]. Leakage quantification methods could help to detect, quantify and minimise those displacement effects even alongside a national accounting system, in order to enhance effectiveness and efficiency of REDD activities. Secondly, due 
to large differences in national circumstances and MRV capacities [14], it might take several years for most REDD countries to develop full-fledged national scale inventory and accounting systems. For any REDD project activity that is conducted before that, be it within the scope of one of the REDD-pilot initiatives, in future regional compliance schemes such as the California Cap-and-Trade Program, or within the voluntary carbon market, leakage quantification methods are indispensable.

With this assessment we intend to answer the following questions:

(1) Which aspects of leakage are the presently used leakage quantification methods able to assess in terms of type of leakage, tools used for quantification and geographical coverage?

(2) How do these methods match the anticipated pre-requisites of a global REDD mechanism?

The most common technical approach to address leakage on the ground is at present to first minimize the leakage risk as far as possible through careful implementation design that targets local deforestation drivers, and the exclusion of leakage-inducing activities [13,15,16], and then quantify and discount the remaining leakages from the overall climate benefits with the help of predefined methods [17]. At present, the most detailed technical specifications of how leakage is quantified are contained in leakage quantification methods that have been developed in the carbon markets under different carbon accounting standards. Another source for leakage quantification methods is the scientific literature, with a range of articles presenting carbon leakage studies. Leakage quantification methods from these two sources are subject to our assessment. Our main focus was on leakage quantification in forest conservation activities. However, due to the relatively young history of forest conservation activities in the carbon market and the scope of REDD having broadened to include enhancement of forest stocks and improved forest management, we also assessed leakage quantification methods from afforestation and reforestation (A/R) and improved forest management (IFM) activities.

Subject to our assessment were leakage quantification methods that are part of carbon accounting methodologies approved under different carbon accounting standards. With carbon accounting standard we refer to the different carbon project standards in the voluntary and regulatory carbon market that allow for forest carbon activities, such as the Verified Carbon Standard (VCS), or the Clean Development Mechanism (CDM) of the Kyoto Protocol. These standards count with one or several carbon accounting methodologies for emission reduction projects in the forest sector, which are accounting guidelines for the step-wise measurement, quantification and monitoring of emission reductions or removals. Our assessment focuses on those sections of the methodologies that specify procedures to estimate leakage emissions-the so-called leakage quantification methods (called "leakage methods" hereafter). In cases where the accounting standards in addition to accounting methodologies also provide stand-alone methodological leakage quantification tools that specifically describe how to quantify leakage from standardized activities (i.e., CDM A/R methodological tools or VCS leakage modules), those were also included in the assessment.

\section{Leakage Analysis Framework}

Leakage effects are not necessarily negative in the sense that they lead to more emissions [9]. When emission reduction activities motivate emission savings outside the accounting boundary, these positive effects are more commonly referred to as spillover effects or benign leakage [6,8]. Positive 
leakage effects are usually not considered in present-day emissions accounting and are thus not covered in this paper. Negative leakage has the potential to significantly undermine or even outweigh the net climate benefits of emission reduction activities [18]. In order to ensure the environmental integrity and effectiveness of climate action, it is thus essential to address and capture leakage effects of emission reduction activities.

Leakage was one of the main methodological concerns why avoided deforestation was excluded from the CDM's eligible land-use options in 2001 [4,19]. Since concerns about leakage came up in the policy negotiations surrounding the role of the land use sector in the Kyoto Protocol, the scientific literature has addressed the topic on conceptual and methodological levels. The resulting literature establishes key definitions and categories that can be used as a framework for analysis [20]. This framework includes two main characteristics of leakage, which serve as basis for our assessment: the geographical scale on which leakage occurs and the type of leakage (direct or indirect, or primary or secondary). In addition, this framework of analysis can be extended with the type of tools that are used for detecting, measuring and quantifying leakage effects.

\subsection{Geographical Leakage Scale}

Leakage is likely to occur when the scale of intervention or regulation is smaller than the scale of the overall problem [17]; making it in principle an accounting issue [21]: Emissions leakage would not exist if all countries were subject to emissions budgeting. In that case, displacement of activities and emissions could still occur, but it would be captured within the global emissions accounting system and thus no longer be defined as leakage. Leakage is defined as displacement to outside the accounting system that can easily go undetected, while displacement within the system will automatically be reflected in emissions balances. Although detected, it nevertheless still reduces the effectiveness (and cost-efficiency) of emission reduction policies. Emissions displacement can take place on all geographical levels and scales [17], depending on the drivers of deforestation, in this case called leakage drivers. Leakage can be a localized process, mainly when smallholders or local communities are affected in subsistence activities such as small-scale agriculture or firewood collection. It can also be a further reaching process, for example when international players and/or the production of market commodities or are affected. These different scales are reflected in the geographical leakage categories described by Schwarze et al. [9], which distinguish local, regional, national and international leakage. While displacement on a local, regional and national scale is clearly the responsibility of the country in which it occurs, international leakage is particularly hard to account for as it is difficult to accurately attribute increasing emissions (or deforestation) in one country to emissions regulation (or forest conservation) in another country [4,10].

\subsection{Leakage Types}

Leakage on all geographical levels can be distinguished by the nature of the processes that motivate emissions displacement. Auckland et al. [15] describe two main categories or types of leakage: primary and secondary. Primary leakage refers to direct leakage effects caused by displacement of baseline activities or agents from one area to the next [15]. Also referred to as "activity shifting” [9], primary leakage occurs when an emission reduction activity limits the supply of goods and services 
that people depend upon in order to meet the continued demand the activities shift to elsewhere. Typical examples are forest conservation activities that reduce deforestation from subsistence production, e.g., shifting cultivation or fuel wood gathering. In order to produce the agricultural crops or obtain the fuel wood needed, local deforestation agents are likely to move to surrounding areas to continue activities. In cases where deforestation agents are internationally operating logging or agribusiness companies, primary leakage can also occur on an international level and be driven by commercial motives.

Secondary leakage occurs when forest conservation in one place indirectly creates incentives to deforest in other places [15]. These indirect effects are usually caused by the reduction in supply of commercial products (e.g., timber), which lead to a shift in market equilibrium. An example would be the reduction of forest activities that produce large quantities of goods that are traded in domestic or international markets, where a reduction in supply could lead to higher prices for these goods. In order to regain the market equilibrium and meet existing demand, production (e.g., logging) shifts elsewhere. The difference to primary leakage is that the forest conservation activity causes incentives for others to start deforesting, rather than encourage deforestation agents to move to new places. These changes can usually be estimated rather than observed directly, thus requiring indirect tools such as economic analyses and modeling instead of direct measurements for quantification [7]. This type of leakage is often referred to as "market effects" [9], or, when linked to the biofuel discussion, "indirect land use change” [22]. It is most likely to occur on a national and international scale.

Schwarze et al. [9] distinguish ecological leakage as a third leakage type, referring to natural processes inside a project's boundary that cause changes in emissions fluxes in surrounding ecosystems. An example would be peatland projects that change the water table within the project area, leading to a change in carbon fluxes both within and outside the project accounting boundary. As this leakage type is not relevant for forest conservation, it is beyond the scope of this paper.

\subsection{Leakage Identification Tools}

Different ways exist to detect and measure leakage effects, both directly and indirectly. Primary leakage from activity shifting can best be detected with the help of direct measurements, often in a reference area established around a project where activities are likely to be displaced to in case leakage occurs. Measurement tools to quantify leakage effects in the reference area include for example remote sensing or ground measurements of forest area, biomass and carbon stocks, as well as interviews and household surveys in local communities that supply information about displaced activities and their magnitude. Tools used for quantifying secondary leakage are for example economic market models, which try to predict changes in market equilibrium and the resulting leakage effects, or the use of default discount factors to the achieved emission reductions in order to account for market effects without directly quantifying them. Different leakage quantification tools can show large variations in the type and quality of data required and the accuracy of estimates they provide.

The assessment presented in this paper is based on the characteristics of leakage described in the framework above:

(1) The leakage type covered by the method: primary or secondary, 
(2) The tool used for data collection required by the quantification method: area measurements or household surveys in the field, direct monitoring of deforestation agents, modelling, or the use of a default leakage factor, and

(3) The geographical leakage scale: local, regional, national or international.

\section{Materials and Methods}

The methodological approach is based on the three characteristics of leakage type, tool and geographical scale presented above and consists of three different steps.

\subsection{Literature Review on the Role of Leakage in REDD}

As a first step, we conducted a literature review on leakage in REDD in order to identify specific requirements for leakage methods in a REDD mechanism. To that end, a literature search in Scopus and ISI Web of Science was conducted to obtain scientific publications on leakage from forest conservation activities and REDD. The identified articles were screened for information on the role of leakage and the requirements of its quantification in forest conservation and REDD, as well as for leakage magnitude estimates. Based on the three main characteristics of the conceptual leakage framework described in Section 2, a set of key features was identified that are important for leakage quantification methods in order to appropriately account for leakage from REDD.

\subsection{Identification of Empirical Material for Assessment}

The identification of empirical material for assessment consisted of a targeted literature search to identify leakage quantification methods described in peer-reviewed scientific journals on the one hand and of the identification of carbon accounting standards for forest carbon activities that involve quantitative leakage methods on the other.

\subsubsection{Leakage Methods Described in the Scientific Literature}

A second literature search was conducted in order to identify leakage methods described in peer-reviewed scientific publications as empirical material for our assessment. We screened the electronic reference resources Scopus and the ISI Web of Science for peer-reviewed articles in English with the search terms "leakage" in the title and "forest*" in the topic (ISI) or in "Title, Abstract, Keywords” (Scopus). The search was conducted for the period of 1975 to 2011, as 1975 is the earliest year that Web of Science provides information about. The search resulted in 28 hits in ISI and 31 hits in Scopus. The abstracts of these articles were screened to identify those that describe methods to determine leakage from forest carbon activities. The main criteria for selection were that a quantitative method was presented, thus excluding qualitative and analytical approaches, and that carbon leakage was explicitly addressed. This resulted in a final list of eight quantitative leakage quantification methods, four addressing secondary leakage [18,23-25] and four addressing primary leakage [26-29]. (Detailed bibliography and description in the Electronic Supplementary Information, ESI 1). We are aware that the narrow focus on carbon leakage somewhat limited the scope of our assessment and led to the exclusion of methods without a clear link to emissions, which might have provided valuable 
insights into methodological approaches to displacement. Such methods include for example modelling exercises that estimate timber market effects (e.g., Sohngen et al. 1999, 2000; Wear and Murray 2004, Zhang and Gan 2007 [30-33]), as well as studies on displacement of deforestation following forest transition (e.g., Meyfroidt et al. 2009, 2010 [34,35]).

\subsubsection{Leakage Methods Approved under Carbon Accounting Standards}

In addition to the leakage methods presented in the scientific literature, the currently most widely used carbon accounting standards in the forestry sector of the global carbon markets were assessed as of their status in June 2011. First, the most prominent carbon accounting standards allowing for forest and land-based activities were identified from literature [36,37] and carbon market statistics [38,39]. The identified forest carbon accounting standards were: the Kyoto Protocol's Clean Development Mechanism (CDM), the Verified Carbon Standard (VCS), the Climate Community and Biodiversity Standards (CCB), the REDD+ Social \& Environmental Standards, Plan Vivo, the US Climate Action Reserve (CAR), and the CarbonFix Standard (CFS). We then restricted our assessment to those standards that (a) require quantitative methods to determine leakage, (b) provide or approve GHG accounting methodologies, and (c) include issuance of carbon credits. Based on these criteria, the standards selected as empirical basis for our assessment are: the CDM, CAR, the VCS, and the CFS [40-43]. We then collected all carbon accounting methodologies for REDD, improved forest management (IFM) and afforestation and reforestation (A/R) activities that have been approved under these standards until June 2011, and identified the respective leakage quantification methods described in these. The full list of carbon accounting standards and leakage methods selected for our assessment is contained in the Electronic Supplementary Information (ESI 2).

Eventually, the assessment covers a total of 34 leakage quantification methods:

- 12 from the CDM: $10 \mathrm{~A} / \mathrm{R}$ methodologies and 2 leakage quantification tools,

- 12 from the VCS: 8 methodologies for REDD and IFM and 4 leakage modules,

- 1 from the CAR: Forestry Protocol Version 3.2,

- 1 from the Carbon Fix Standard v 3.1,

- 8 methods or case studies for leakage quantification presented in peer-reviewed scientific articles [18,23-29].

\subsection{Analysis of Leakage Quantification Methods}

The leakage methods identified in the steps above formed the empirical material for our assessment. A first screening of methods for the three basic characteristics they use to addressing leakage (leakage type, quantification tool used, and geographical scale covered, see characteristics 1-3 in Section 2) showed that they can be grouped into nine main methodological approaches based on which leakage characteristics they address. We then assessed these nine methodological approaches for their applicability in a REDD mechanism by comparing them to the key features for leakage quantification in REDD that were identified during the literature review. In a final step we determined which of the main methodological approaches are used in the four carbon accounting standards in order to find out which of those is best prepared to address leakage in a future REDD mechanism. 


\section{Results and Discussion}

\subsection{Literature Review: The Role of Leakage in REDD}

Leakage in REDD is considered by several authors a challenging technical issue with the potential to negatively impact environmental integrity and effectiveness of the mechanism [21,25,44]; in terms of effectively reducing both emissions and global deforestation rates. Financial incentives from REDD to keep forest in place might be high enough to compensate for foregone revenues from low-income land use activities, thus covering the opportunity cost of land [45]. However, if local subsistence production is jeopardised through REDD activities, substantial activity shifting leakage could take place on a local scale [13], thus making primary leakage a central aspect to consider in REDD. Fisher et al. [13] therefore consider REDD measures that directly address those local drivers as key to reduce the risk for leakage. In addition revenue-strong commercial land use options such as cash crops or cattle breeding as well as the selling of timber can produce lucrative incomes and thus be a strong incentive to deforest. Under a REDD mechanism, producers of commodities such as palm oil, timber or beef could simply shift to regions that do not participate in REDD [10,16,46], thus creating further primary leakage. Alternatively or even in parallel, a continued international demand for internationally traded commodities could lead to higher prices when meeting a reduced production in REDD countries; thus incentivising production elsewhere and creating market leakage [44]. The latter two cases suggest a high potential for international leakage, which is supported by literature findings: Gan and McCarl [18] for example find international leakage to be higher than $65 \%$ for all countries except Canada if there is no global cooperation in forest conservation efforts. Several market leakage estimates for forest conservation activities on national and international scales [18,23-25] indicate that secondary leakage could reduce climate effectiveness of forest conservation activities by 19-64\% (taking average values of the ranges in Table 1), with national leakage effects ranging between 0 and 92\%, and international leakage between 42 and 95\% (Table 1). While moderate leakage levels might be counter-productive but tolerable from an efficiency point of view, leakage effects of more than $50 \%$ represent a serious limitation to the cost-efficiency of forest conservation measures, and can make them economically non-viable.

Table 1. Different scientific publications estimating leakage effects of forest conservation activities on regional, national or global scale. Average indicates the average of all low vs. all high values. Squared brackets indicate the reference as presented in the reference list.

\begin{tabular}{llll}
\hline Authors & Activity causing leakage & $\begin{array}{l}\text { Scale of leakage } \\
\text { assessment }\end{array}$ & Magnitude \\
\hline $\begin{array}{l}\text { [18] Gan and McCarl (2007) } \\
\text { [23] Sun and Sohngen (2009) }\end{array}$ & $\begin{array}{l}\text { Forest conservation } \\
\text { Forest conservation, improved forest } \\
\text { management, afforestation }\end{array}$ & Global & $42-95 \%$ \\
[24] Sohngen and Brown & Forest conservation & National (Bolivia) & 5-42\% \\
(2004) & & & (2-38\% discounted) \\
[25] Murray et al. (2004) & $\begin{array}{l}\text { Forest conservation, improved forest } \\
\text { management, afforestation }\end{array}$ & National (US) & 0-92\% \\
& & Average & $\mathbf{1 9 - 6 4 \%}$ \\
\hline
\end{tabular}


Although they cannot be completely eliminated, several authors agree that REDD leakage effects on a national scale can be managed, quantified and eventually subtracted from overall emission reductions achieved [7,17,25]. This does not apply for international leakage, which is considered a major challenge both for REDD and other mitigation sectors [21,44].

While in the long run within-country leakage would be accounted for in national inventory systems, international leakage in REDD could be "rampant" as described by Skutsch and McCarl [44]. While accounting for international leakage is not required in the present emissions accounting framework, it is still beneficial for the sake of environmental integrity to identify international displacement effects and their magnitudes, not only for REDD but in all GHG emission sectors. It is therefore essential to effectively and comprehensively capture leakage in order to address and minimize the leakage drivers as well as quantify and account for unavoidable leakage effects. To this end, we have identified three key features from the literature in line with the characteristics of the leakage analysis framework, which we consider essential for effective REDD leakage methods (Table 2).

Table 2. Features that leakage quantification methods for REDD should fulfill, based on the three characteristics of the leakage analysis framework (leakage type, quantification tool and geographical scale covered).

\begin{tabular}{|c|c|c|}
\hline $\begin{array}{c}\text { Leakage } \\
\text { characteristic }\end{array}$ & Description of leakage characteristic in REDD literature & $\begin{array}{c}\text { Recommended method } \\
\text { feature } \\
\end{array}$ \\
\hline Leakage type & $\begin{array}{l}\text { Leakage both from activity shifting (primary) and market } \\
\text { effects (secondary) need to be covered to ensure that all displacement } \\
\text { effects are comprehensively captured [47]. }\end{array}$ & $\begin{array}{l}\text { I. Leakage methods for } \\
\text { REDD should thus address } \\
\text { all relevant types of } \\
\text { leakage, direct and indirect }\end{array}$ \\
\hline $\begin{array}{l}\text { Quantification } \\
\text { tool }\end{array}$ & $\begin{array}{l}\text { To effectively capture all types of leakage, a range of appropriate tools } \\
\text { for quantifying both primary and secondary leakage effects is required. } \\
\text { Appropriate tools in this context are: } \\
\text { (a) for primary leakage: based on measurements or interviews in control } \\
\text { areas [17] or direct monitoring where possible; if estimates are } \\
\text { unavoidable these should be conservative [8]; leakage effects should be } \\
\text { subsequently monitored at least in the project surrounding. } \\
\text { (b) for secondary leakage: market indicators used in modelling should } \\
\text { be monitored and adjusted when needed [10]; discount factors should } \\
\text { depend on project conditions; i.e., be dynamic rather than generic }\end{array}$ & $\begin{array}{l}\text { II. Leakage methods for } \\
\text { REDD should thus cover a } \\
\text { range of appropriate } \\
\text { assessment tools. }\end{array}$ \\
\hline $\begin{array}{l}\text { Geographical } \\
\text { scale }\end{array}$ & $\begin{array}{l}\text { In order to comprehensively address leakage, emissions displacement } \\
\text { on all scales has to be captured: localised effects need to be measured } \\
\text { on local and regional scale, while } \\
\text { wide-ranging effects require detection and monitoring on national and } \\
\text { international scale }[11,12] \text {. }\end{array}$ & $\begin{array}{l}\text { III. For comprehensive } \\
\text { leakage accounting, } \\
\text { eventually on national-scale } \\
\text { as requested by the } \\
\text { UNFCCC [11], REDD } \\
\text { leakage methods should } \\
\text { capture leakage effects on } \\
\text { at least national scale. }\end{array}$ \\
\hline
\end{tabular}




\subsection{Assessment of Leakage Quantification Methods}

A screening of leakage methods against the three basic characteristics of the analysis framework (leakage type, quantification tool, and geographical scale covered) shows that some of the methods take similar approaches to quantifying leakage, which allows grouping them into nine main methodological approaches. These nine approaches can be further divided according to the leakage type they address, since methods addressing primary leakage show common features as well as methods addressing secondary leakage. This yields a group of six methodological approaches to primary leakage (Primary Leakage Approach, PLA 1-6), and three to secondary leakage (Secondary Leakage Approach, SLA 1-3). Figure 1 below illustrates how the 34 assessed leakage quantification methods can be grouped into each of the main methodological approaches. The sum of methods exceeds 34 in the figure; the difference arises because some of the 34 assessed leakage methods belong to several main approaches; e.g., the VCS IFM methods that address both primary and secondary leakage.

Figure 1. Leakage quantification methods applying the nine main methodological approaches. PLA-Primary Leakage Approach, SLA-Secondary Leakage Approach; CDM—Clean Development Mechanism, VCS—Verified Carbon Standard, CAR—Climate Action Reserve, CFS—Carbon Fix Standard.

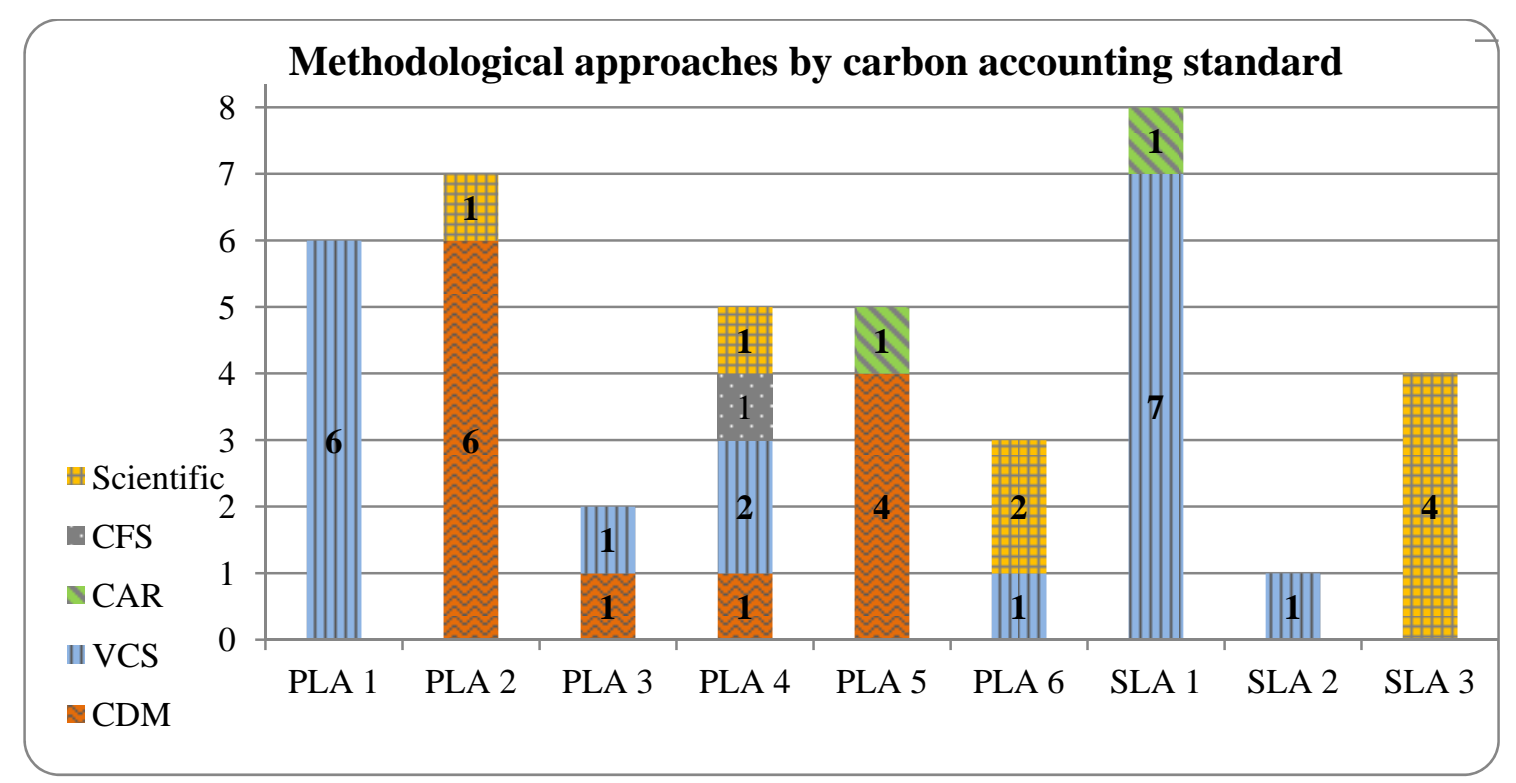

\subsubsection{Methods to Quantify Primary Leakage}

The choice of method to quantify primary leakage depends on the activity that is displaced when protecting forests or establishing new forests. When a project prevents planned deforestation or degradation (e.g., for land conversion or logging), and the deforestation agent is known, leakage methods are usually straightforward and involve direct monitoring of shifting activities. In cases where deforestation happens in an unplanned manner (e.g., through subsistence agriculture, logging or fuel wood collection), methods to detect leakage are more complex and involve different measurement and deduction steps. 
Primary Leakage Approach 1: Leakage from the avoidance of planned and legally authorized logging activities. Where leakage is caused by a shifting of planned activities, for example in the case of avoiding logging in a legally obtained concession, the deforestation agent is easily identified and his actions can be monitored over time. Six of seven VCS IFM methodologies use this approach, which covers the national scale. Activity shifting in this approach is addressed by examining logging and harvest volumes of all lands under the forest owner's control, and establishing a historical average that reaches at least 5 years into the past. This historical baseline is compared to the monitored actual harvest levels in all forest areas outside the project area controlled by the project developer, and if the latter is higher than the former, the difference between actual and baseline levels is considered leakage. In order to quantify leakage emissions, the increased timber volumes logged are converted to carbon and CO2. This approach also works in cases where the deforestation agent is not a physical person, but a government agency or corporation. In case the deforestation agent cannot be clearly identified, and only a class of agents (e.g., palm oil producers) can be established, leakage is either determined through the difference between historic and with-project rates of deforestation caused by the class of agents within the region, or it is linked to market demand for the commodity whose production is prevented by the project, thus applying market leakage quantification methods.

Primary Leakage Approach 2: Leakage from agricultural activities (unplanned deforestation). This leakage approach is most common in reforestation projects (CDM and CFS) that restrict non-commercial agricultural activities in the project area. The quantification of leakage from the relocation of agricultural activities is based on tracking and monitoring the activities that are displaced to outside the project boundary. Leakage emissions are quantified by combining the project-induced increase in agricultural area outside the project area with the carbon stock depleted by the increase. For measurement purposes, usually a reference area is established around the project in which all land use changes are monitored through remote sensing and/or measurements on the ground. The latter can be field measurements of the actual areas covered by cropland or grazing land inside and outside the project area before and after project start to identify any leakage-induced increase in the area. Another way to gather this information is to conduct interviews, household surveys or participatory rural appraisals (PRA [48]) with all or representative samples of people that practice agriculture in and outside the project area before the project starts. Based on these data, ex-ante estimates of leakage are established which are later monitored and adjusted for field measurements of the actual leakage extent. This approach assumes that land use activities will be displaced to neighboring areas only, and thus has a local geographical cover. Four CDM A/R methodologies and the CDM Leakage Tool for Agriculture and Grazing [40] use this approach. The peer-reviewed method presented by Ewers and Rodrigues [26] partly applies this approach through measurements of deforestation in a reference area. Their method differs slightly because it then compares expected deforestation rates based on a landscape-wide baseline with the observed deforestation. Leakage then equals the fraction of observed deforestation that exceeds the expected deforestation rate.

Primary Leakage Approach 3: Leakage from fuel wood collection (unplanned forest degradation). Typically, fuel wood collection leads to forest degradation rather than deforestation, and only in cases where the fuel wood is collected in an unsustainable way. The CDM Leakage tool for non-renewable biomass [40] uses a simple approach to determine leakage by measuring the increase in fuel wood collection outside the project area over the baseline conditions (in wood volume or mass). Volumes of 
fuel wood used inside and outside the project area are obtained through community interviews, field measurements and/or literature data. Only the fraction of the increase that is harvested unsustainably, i.e., constitutes non-renewable biomass (as defined by the CDM Executive Board [49]) is considered to cause leakage. According to that definition, biomass is non-renewable when its extraction leads to a land use change or when carbon stock is systematically decreased over time, or when fuel wood collection is against national law. The leakage emissions from non-renewable fuel wood use are equal to the carbon contained in the biomass that is lost and not renewed. Due to its focus on the project area and close surroundings, the geographical level covered by this approach is local. While none of the CDM A/R methodologies approved so far uses this tool, it is applied in the VCS methodological module on fuel-wood leakage.

Primary Leakage Approach 4: Leakage from agriculture, fuel wood collection and other leakage drivers. This leakage approach provides an extension to PLA 2 by (a) covering more leakage drivers, such as leakage from agriculture, fuel wood collection, settlements and forest fires (b) differentiating between activities displaced to the direct project surrounding, and activities that migrate to other locations in the country. The latter are divided into identifiable and non-identifiable emissions displacement processes, by some methods also called geographically constrained and un-constrained leakage drivers: locally constrained, identifiable leakage means displacement from inside the project area to its close surroundings, which can be directly identified and quantified as per PLA 2.

Locally unconstrained and non-identifiable leakage occurs in a region out of the project's direct reach. This can be for example when livestock is sold, or when the immediate area around the project does not have sufficient carrying capacity to sustain the displaced activities in addition to existing ones. This makes it impossible to determine how much carbon stock is depleted through the shift of activities from unconstrained drivers; therefore this approach uses an indirect way to account for eventual leakage from migrating activities. The conservative assumption is used that an average regional or national forest (with average biomass and carbon content) is fully converted to non-forest by the proportion of activities that is displaced to non-identifiable areas. This proportion is determined by subtracting the observed activity shifting from inside to outside of the project area from the total displaced land use activities to outside the project area. The VCS methodologies applying this approach allow for an adjustment of this $100 \%$ leakage assumption in case a smaller leakage factor can be credibly demonstrated. Through capturing effects that migrate out of the direct project surrounding to other regions in the country, this leakage approach reaches up to the national scale and covers leakage effects within the country. One CDM and two VCS methodologies use this approach, as well as the peer-reviewed method described by Dutschke et al. [27]. The latter develops the PARAPIA approach that uses a direct reference area around the project to measure geographically constrained leakage, and estimates geographically unconstrained leakage due to the persons migrating to outside the reference area based on the national average emissions per capita. Another methodology that uses this leakage approach with slight adjustments is the Carbon Fix Standard [43] when the area that activities are displaced to is not known, the emissions are either based on the carbon stock of the original project area or the average carbon stock of a natural forest within the country.

Primary Leakage Approach 5: Simplified factor approach to leakage from agriculture and fuelwood collection. A simplified approach that does not require tracking and monitoring of activities outside the project area involves the use of leakage discount values depending on the agriculture and 
fuel-wood activities in the project area that are displaced. Agricultural activities and fuel wood collection are thus exclusively measured and monitored within the project area, and if a pre-defined area threshold of activity shifting is exceeded (supported by measurements of area and activity changes within the project area), the application of generic leakage discounts is triggered. This approach is used in four CDM methodologies and the CAR Forest Protocol [50]. Two of the CDM methodologies use a threshold for cropland area displaced: if it exceeds $10 \%$ of the total project area, a $15 \%$ leakage discount is applied to the GHG removals achieved by the project. Another CDM methodology assigns a leakage discount of $20 \%$ if the cropland area displaced is lower than $10 \%$ of total project area; and if leakage from fuel-wood collection cannot be excluded, a 5\% discount applies. A CDM large scale methodology requires a generic $10 \%$ leakage discount on the carbon content of the baseline scenario when leakage from fuel-wood collection cannot be excluded. The CAR leakage methodology for reforestation and avoided conversion projects also belongs in this category. The leakage discounts used by CAR depend on conditions within the project area; e.g., reforestation projects account for leakage from combustion in site preparation based on the amount of vegetation on site that has to be cleared; depending on the ground cover ( 25 to $>50 \%$ ), the leakage discount varies between 9 and $43 \%$. Leakage discounts for displacement of grazing vary with the planned project canopy cover of reforestation projects; a cover of $>30 \%$ is expected to cause leakage as grazing is hampered in denser forests. The leakage discount used increases stepwise from 10-50\% with increasing canopy cover, while reforestation on commercially viable cropland triggers a generic discount of $24 \%$ for leakage from agriculture. Leakage discounts from reforestation are determined once before project start and not monitored afterwards. Only forest conversion (=REDD) projects require subsequent measurements, as they apply a generic discount of 3.6\% if the decrease in carbon stock in the project is higher than in the baseline scenario.

Primary Leakage Approach 6: Modeling of activity shifting leakage. Three of the assessed methods use modeling approaches to primary leakage. VCS VM0009 uses a combination of model predictions and on-the-ground observations in a leakage reference area to determine a leakage factor. The model specifies the expected baseline deforestation and degradation in the leakage reference area in the absence of the project. These predictions are then compared to deforestation observed on the ground, and if deforestation rates are higher in reality than predicted by the model, leakage has occurred. Based on the difference between the leakage model rates and observed rates, a leakage factor is determined which is applied to the baseline emissions.

The peer-reviewed method by Boer et al. [28] uses a logistic model to estimate the probability of a land use being converted to other uses. They use several predictor variables such as proximity to the project area, distance to transportation channel, area of agricultural land, employment situation, population density and income to establish probabilities of land use change. These probabilities of forest conversion to agricultural land are modeled in two reforestation scenarios and a baseline scenario within a regional district.

Lasco et al. [29] use an approach similar to this logistic model, assuming that leakage can be calculated as a function of technology adoption rates that local communities show to alternative livelihoods. Historical adoption rates for agroforestry activities in the Philippines are used to establish a reference scenario: the percentage of population that does not quickly adopt new technologies is 
expected to cause leakage effects as they stick to traditional land use activities. All three modeling approaches to primary leakage cover the local and regional, but not the national scale.

\subsubsection{Methods to Quantify Secondary Leakage}

Secondary leakage methods by definition use indirect quantification approaches, as market effects cannot be directly observed and measured. The identified approaches differ in the choice of indirect method; the majority (8 of 12) use discounting of achieved project emission reductions based on more or less generic leakage factors, while four are based on market modeling.

Secondary Leakage Approach 1: Discount factor approach to market leakage. Eight methods that account for leakage from market effects use a leakage discount factor approach, which accounts for market leakage effects without being able to directly quantify them. Market leakage emissions are accounted for by multiplying the net change in carbon stock with a discount factor, which can have values between 0 and 1 . The details of how this approach is applied can vary between project types and carbon standards. For VCS IFM projects the leakage discount depends on the biomass conditions of the forests where logging will be increased as result of the decreased timber supply caused by the project. The reasoning behind is that if the new area has a lower merchantable biomass per hectare than the project area, additional logging will be needed to compensate this reduction and leakage emissions would be higher. In contrast, if the new area shows higher merchantable biomass per hectare, leakage would be lower because it is possible to meet the demand for wood with less hectares affected by logging.

In order to determine the leakage factor, the (commercial) biomass content is compared in the project area and the forest area where harvesting will most likely be displaced to. The VCS approach specifies leakage factors of $0.4(40 \%)$ if the carbon content in both areas is equal, of 0.7 (70\%) if the content in the new areas is lower, and 0.2 (20\%) if the content in the new area is higher than in the project area.

This factor approach can in adjusted form be used for other types of decreased harvest, e.g., in one method that deals with decreased harvest of fuel wood and charcoal making. The parameters used to determine the leakage factor are then not commercial biomass density but the suitability of displacement forests for charcoal making and fuel wood. This leakage factor approach is used by seven VCS methods, with two of these providing slight adjustments [51]. In addition to this approach, a simplified leakage factor approach is used by the CAR Forest Protocol, which specifies a generic leakage market factor of 0.2 (20\%) to net emission reductions achieved by IFM projects. The use of the CAR approach is also endorsed by the VCS methodology VM0012 for VCS projects in the CAR region - the US, Canada, Brazil and Mexico.

Secondary Leakage Approach 2: Detailed market assessment. The VCS methodology VM0012 develops a detailed market leakage assessment tool that is based on SLA1 but differentiates leakage further through weighting leakage effects into different biomass-density classes, and into national and international leakage effects. SLA1 assumes conservatively that all leakage remains in country, while the tool described in VM0012 requires an assessment of leakage risk based on forest product markets data. With the help of this, the fraction of leakage that is expected to be replaced by international sources is estimated and can be accounted for as zero, as specified by VCS requirements. This 
approach follows the same logic as PLA 3 that distinguishes geographically constrained and unconstrained leakage; the constrained leakage being in this case the fraction remaining in country, and the unconstrained the fraction that leaves the country. However, only the fraction of leakage remaining in country needs to be quantified and be accounted for under current accounting rules.

Secondary Leakage Approach 3: Modeling of market leakage. While the above approaches account for market leakage effects through fix discount percentages, other methods using market modeling yield more detailed leakage estimates. Although the VCS (as only carbon standard) mentions market modeling as one possible tool to account for market leakage, none of the VCS methodologies makes use of this option. However the four peer-reviewed secondary leakage methods from the scientific literature use equilibrium models to quantify market effects. These models operate under the assumption of ideal conditions of market equilibrium and perfect competition, and optimize net present values of consumer and producer surplus. Gan and McCarl [18] estimate international displacement effects from forest conservation with the general equilibrium model GTAP. Sun and Sohngen [23] model global leakage effects from three different set-aside scenarios with a global land use and forestry model. Murray et al. [25] use the forest and agriculture sector model FASOM to estimate leakage from different forest carbon sequestration activities on a national scale in the US. Sohngen and Brown [24] develop a dynamic timber market optimization model to determine national-scale leakage effects of forest conservation in Bolivia. The latter two methods are recommended by the VCS as modeling tools if projects choose the modeling of secondary leakage over the discount approach. All models used in this approach yield detailed estimates of leakage effects, which, however, depend strongly on the scenarios and input data used. Depending on the type of market data used, the market models can cover national or international scales. The modeling approaches require large amounts of input data, which could be one of the reasons why in our assessment they were exclusively found in the scientific literature. The high demand for input data together with often restricted data availability makes the use of equilibrium models in the rather pragmatic carbon accounting standards difficult.

Based on the description of these methodological approaches, Table 3 summarizes the findings of screening the different approaches against the basic leakage characteristics type, quantification tool, and geographical scale.

\subsection{How the Carbon Accounting Standards Address Leakage}

In a next step we assess how the different carbon accounting standards make use of the different methodological approaches, discussing the appropriateness of leakage accounting within the standards. Figure 1 above illustrates which standards use which methodological approaches, while a detailed overview of which methods apply to which approaches is found in the Electronic Supplementary Information ESI 3. 
Table 3. Results of screening the methodological approaches for the basic leakage characteristics 1-3. PLA-Primary Leakage Approaches, SLA-Secondary Leakage Approaches. Squared brackets indicate tools that are used by a method without being the main quantification tool.

\begin{tabular}{|c|c|c|c|c|c|c|c|c|c|c|c|c|}
\hline \multirow[b]{2}{*}{$\begin{array}{l}\text { Metho- } \\
\text { dological } \\
\text { approach }\end{array}$} & \multirow[b]{2}{*}{ Methods using the approach } & \multicolumn{2}{|c|}{$\begin{array}{c}\text { Leakage } \\
\text { type }\end{array}$} & \multicolumn{5}{|c|}{ Tool for quantification } & \multicolumn{4}{|c|}{ Geographical scale } \\
\hline & & $\begin{array}{l}\vec{\Xi} \\
\text { 泀 } \\
\end{array}$ & 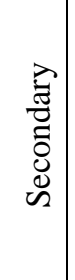 & 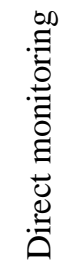 & 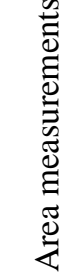 & 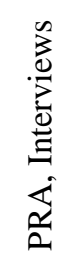 & 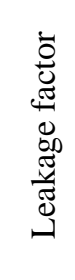 & 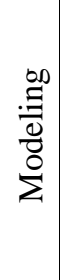 & $\begin{array}{l}\overline{0} \\
0 \\
0\end{array}$ & $\begin{array}{l}\overline{\widetilde{J}} \\
\overline{\tilde{7}} \\
\bar{\pi}\end{array}$ & 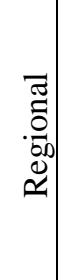 & ]్ర్ \\
\hline PLA 1 & $\begin{array}{l}\text { VCS: VM0003, VM0004, } \\
\text { VM0010; VM0011, VM0012, } \\
\text { VMD009 }\end{array}$ & $\mathrm{x}$ & & $\mathrm{x}$ & & & & & & $\mathrm{x}$ & $\mathrm{x}$ & $\mathrm{x}$ \\
\hline PLA 2 & $\begin{array}{l}\text { CDM: AR-AM0005; } \\
\text { AR-AM0011, AR-AM0013, } \\
\text { AR-CM 0001, CDM LK tool } \\
\text { for Agriculture; Ewers } \\
\text { \& Rodrigues [26] }\end{array}$ & $\mathrm{x}$ & & $\mathrm{x}$ & $\mathrm{x}$ & $\mathrm{x}$ & & & & & & $\mathrm{x}$ \\
\hline PLA 3 & $\begin{array}{l}\text { CDM: Leakage tool for } \\
\text { non-renewable biomass, VCS: } \\
\text { VMD0012 }\end{array}$ & $\mathrm{x}$ & & & $\mathrm{x}$ & $\mathrm{x}$ & & & & & & $x$ \\
\hline PLA 4 & $\begin{array}{l}\text { CDM: AR-AM0004 } \\
\text { VCS: VM0006, VMD0010; } \\
\text { Carbon Fix; } \\
\text { Dutschke et al. [27] }\end{array}$ & $\mathrm{x}$ & & $\mathrm{x}$ & $\mathrm{x}$ & $\mathrm{x}$ & $(\mathrm{x})$ & & & $\mathrm{x}$ & $\mathrm{x}$ & $\mathrm{x}$ \\
\hline PLA 5 & $\begin{array}{l}\text { CDM: AR-AMS } 0001 \\
\text { AR-AMS 0002, AR-AMS } \\
\text { 0003, AR-AM 0014; CAR } \\
\text { Forest Protocol }\end{array}$ & $\mathrm{x}$ & & & $(\mathrm{x})$ & & $\mathrm{x}$ & & & $\mathrm{x}$ & $\mathrm{x}$ & $\mathrm{x}$ \\
\hline PLA 6 & $\begin{array}{l}\text { VCS: VM0009 } \\
\text { Boer et al. [28] } \\
\text { Lasco et al. [29] }\end{array}$ & $\mathrm{x}$ & & & $(\mathrm{x})$ & & & $\mathrm{x}$ & & & $\mathrm{x}$ & $\mathrm{x}$ \\
\hline SLA 1 & $\begin{array}{l}\text { VCS: VM0003,VM0004; } \\
\text { VM0005; VM0010; } \\
\text { VM0011; VM0012; } \\
\text { VMD0011; CAR }\end{array}$ & & $\mathrm{x}$ & & & & $x$ & & & $\mathrm{x}$ & $x$ & $\mathrm{x}$ \\
\hline SLA 2 & VCS: VM0012 & & $\mathrm{x}$ & & & & $\mathrm{x}$ & & $(\mathrm{x}) *$ & $\mathrm{x}$ & $\mathrm{x}$ & $\mathrm{x}$ \\
\hline SLA 3 & $\begin{array}{l}\text { Gan \& McCarl [18] } \\
\text { Sun \& Sohngen [23] } \\
\text { Sohngen \& Brown [24] } \\
\text { Murray et al. [25] }\end{array}$ & & $\mathrm{x}$ & & & & & $\mathrm{x}$ & $\mathrm{x}$ & $\mathrm{x}$ & & \\
\hline
\end{tabular}

* Method has been developed to cover the national scale, but can potentially address the international scale as well. 
CDM. CDM modalities explicitly exclude accounting for market leakage, which is why none of the assessed CDM methods address secondary leakage. CDM leakage methods make use of four of the six primary leakage approaches; PLA 2 (used in 6 cases), PLA 3 (1), PLA 4 (1) and PLA 5 (4). With this, the majority of CDM leakage methods cover the local scale (7 of 12), while only one method comprehensively covers the national scale through consideration of unconstrained leakage (PLA 4). Main tools used in CDM leakage methods are direct area measurements and PRA in project surroundings, as well as conservative assumptions on leakage emissions outside the direct project surroundings. The four methods using generic discounts in principle cover the national scale; however the appropriateness of this approach can be discussed (see 4.3 below). Since these four methods use generic discounts to account for primary leakage, the CDM only partly fulfills the recommended features of comprehensive leakage accounting as described in Table 2 above. Nevertheless, monitoring of leakage is required in all cases.

VCS. The VCS is the only carbon standard that requires accounting for all significant sources of leakage within one country, both primary and secondary [47]. Depending on the project activity, VCS leakage methods can therefore be found in several of the methodological approaches. Primary leakage has to be addressed in all project types; either through demonstration of its absence (IFM projects) or through quantification (REDD projects). In most cases (8 of 12) primary leakage is addressed on national level (through PLA 1 and PLA 4), and in two cases on local level (through PLA 3 and 6). Secondary leakage has to be accounted for in both IFM and REDD projects that significantly reduce the supply of a commercial commodity. Nearly all methods assessed (7 of 8) address market leakage through discounts (SLA 1); in addition one method develops a national-scale market assessment tool (SLA 2); all secondary leakage is assessed on national scale. Although an eligible option, none of the VCS methods chooses to conduct market modeling to determine secondary leakage. Main tools used are direct monitoring of deforestation agents as well as field measurements in reference areas for primary leakage, combined with conservative assumptions for unconstrained leakage to outside the reference area. For secondary leakage, the main tool used is leakage discounts that depend on conditions outside the project area. Subsequent monitoring of leakage is mandatory in all VCS methodologies. With this, the VCS fulfills our definition of using “appropriate tools” specified in Table 2 above.

CAR. The Forestry Protocol v3.2 [41] does not use the categorization of primary and secondary leakage; instead all leakage is summarized in the term "secondary effects". Nevertheless, the Protocol still distinguishes the two leakage types: primary leakage has to be addressed in both reforestation and avoided conversion projects, while forest management projects need to account for secondary leakage from market effects. The standard thus addresses both primary and secondary leakage; however not in combination. Both primary and secondary leakage is accounted for through generic discount factors (PLA 5 and SLA 1). With this approach, CAR leakage accounting covers the national scale. Monitoring of leakage effects is not required by the CAR, however avoided conversion projects need to subsequently measure carbon stock changes in the project area that indicate occurrence of leakage. The generic leakage discount applied to conversion projects is remarkably small (3.6\%) and not further substantiated. With the use of generic discounts for primary leakage, non-project specific discounts for secondary leakage, and the absence of a monitoring requirement the CAR approach to leakage does not capture the complexity of leakage processes from different project conditions, and is thus considered less appropriate according to the definition specified in Table 2. 
Carbon Fix Standard. Similar to the CDM, the leakage type addressed by CFS [43] is activity shifting, while quantification of secondary leakage is not required. The potential drivers of leakage are distinguished through different quantification methods for displacement of fuel-wood collection, charcoal making, logging, farming, grazing or resettlement. CFS' basic approach to leakage considers activity shifting both to the direct project surrounding and to unknown areas, and is thus considered to belong to the comprehensive, national-scale approach PLA4. However, the potential extent of leakage is estimated based on land use change within the project area only, as done in PLA5. The leakage discount to be applied can vary substantially, as it is based either on representative surveys or on credible estimations about the potential percentage of activities to be displaced. Both these concepts are not further defined in the methodology (e.g., what is a credible estimation? what a representative survey?). Beyond an initial monitoring and certification in the first year no monitoring or ex-post quantification of the actual leakage effects is required by the standard. This makes the CFS approach to leakage rather rudimentary.

The scientific literature shows a more complex picture, which does not allow considering the eight peer-reviewed methods as one homogenous group like the carbon accounting standards. Instead, we observe that the scientific methods are very detailed and case-specific, which means that they either address primary or secondary leakage, they apply only one main quantification tool each, and the geographical scale they cover depends on the context. Four of the eight peer-reviewed scientific leakage methods address primary leakage, and four address secondary effects. Two of the methods for primary leakage [28,29] use modeling approaches (PLA6), while one [26] uses PLA 2 and another one [27] PLA4. With this, [26,28,29] target leakage on regional and local scale while [27] covers the national scale. The four methods to quantify secondary leakage all rely on modeling and apply general equilibrium models to quantify leakage on national $[24,25]$ and global $[18,23]$ scales.

Based on these summaries of leakage characteristics addressed by the different carbon accounting standards, Table 4 illustrates how the standards (without scientific literature) address the three basic leakage characteristics.

Table 4. Carbon standards addressing basic leakage characteristics: Leakage type, Quantification tool, Geographical scale. CDM-Clean Development Mechanism, VCSVerified Carbon Standard, CAR—Climate Action Reserve, CFS—Carbon Fix Standard.

\begin{tabular}{|c|c|c|c|c|}
\hline & CDM & VCS & CAR & CFS \\
\hline \multicolumn{5}{|l|}{ Leakage type } \\
\hline Primary & $\mathrm{x}$ & $\mathrm{x}$ & $\mathrm{x}$ & $\mathrm{x}$ \\
\hline Secondary & & $\mathrm{x}$ & $\mathrm{x}$ & \\
\hline \multicolumn{5}{|l|}{ Quantification tool } \\
\hline Direct monitoring & & $\mathrm{x}$ & & \\
\hline Area measurements & $\mathrm{x}$ & $\mathrm{x}$ & & $\mathrm{x}$ \\
\hline PRA, Interviews, Surveys & $\mathrm{x}$ & $\mathrm{x}$ & & $\mathrm{x}$ \\
\hline Leakage factor & $\mathrm{X}$ & $\mathrm{x}$ & $\mathrm{X}$ & \\
\hline Modeling & & $\mathrm{x}$ & & \\
\hline \multicolumn{5}{|l|}{ Leakage scale } \\
\hline Global & & & & \\
\hline National & $\mathrm{x}$ & $\mathrm{x}$ & $\mathrm{x}$ & $\mathrm{x}$ \\
\hline Regional & $\mathrm{x}$ & $\mathrm{x}$ & $\mathrm{x}$ & $\mathrm{x}$ \\
\hline Local & $\mathrm{x}$ & $\mathrm{x}$ & $\mathrm{x}$ & $\mathrm{x}$ \\
\hline
\end{tabular}




\subsection{Do the Methods Assessed Meet the Requirements for Leakage Accounting in REDD?}

The above findings (Tables 3 and 4) describe how the methodological approaches and the carbon accounting standards address the basic characteristics leakage type, quantification tool and geographical scale. These results are in a next step compared with the features that leakage accounting should fulfill in a REDD mechanism determined earlier (see Table 2). In that context it is essential that comprehensive leakage accounting addresses both primary and secondary leakage and that it at least covers the national scale. The tools used to quantify leakage on different geographical scales vary significantly within the methods assessed; therefore it is important that they are appropriate for accurate leakage accounting as defined in Table 2.

\subsubsection{All Relevant Types of Leakage should Be Addressed, both Primary and Secondary}

\subsubsection{Methodological Approaches}

None of the methodological approaches fulfills this requirement per se, as all approaches either address primary or secondary leakage. Leakage in REDD can only be comprehensively addressed if appropriate primary and secondary leakage approaches are combined.

\subsubsection{Carbon Accounting Standards}

While the reforestation standards CDM and CFS are limited to primary leakage, VCS and CAR which allow for forest conservation and improved forest management in addition to reforestation activities account for both primary and secondary leakage. However, the CAR requires either primary or secondary leakage accounting depending on the project activity. The VCS is the only standard that requires combined accounting of both leakage types within one forest carbon activity.

\subsubsection{Leakage Methods for REDD should Cover a Range of Appropriate Assessment Tools}

\subsubsection{Methodological Approaches}

According to our definition of appropriate tools (see Table 2), activity shifting leakage to the project surroundings should be quantified through direct monitoring of agents or through measuring the extension of area change in the field; either with the help of actual ground measurements or interviews. Activity shifting beyond the direct project surrounding is accounted for either through direct monitoring of the deforestation agent if he is known, or indirectly based on conservative assumptions; e.g., assuming that the fraction of leakage that migrates out of the direct project surrounding fully leads to destruction of other forests. Based on this, the primary leakage approaches PLA 1, 2, 3, 4 and 6 in principle fulfill this requirement, as long as they entail monitoring. The use of generic leakage discounts as in PLA 5 is considered too simplistic to provide accurate estimates of leakage effects. As leakage discounts depend on measured parameters within the project area only, leakage effects outside the project boundary are accounted for indirectly rather than directly. This is considered an inappropriate tool for quantifying primary leakage as it is technically possible to directly identify activity shifting effects within the project surrounding through field measurements. Generic leakage discounts should 
be restricted to secondary leakage, which is impossible to measure directly. SLA 1 and 2 partly fulfill the appropriateness-requirement as long as dynamic leakage discounts are used and market information is subsequently updated. The modeling approaches used in SLA 3 are based on theoretical exercises that in principle could be repeated with updated market information, thus being considered appropriate.

\subsubsection{Carbon Accounting Standards}

In relation to the above, carbon standards that use appropriate tools include the VCS and partly the CDM, with the exception of the four simplified methods that use PLA5. The CAR approach to leakage is considered too simplistic to accurately quantify leakage effects. Primary leakage is addressed with PLA 5 and the generic discounts used for REDD projects are very small. Monitoring of primary leakage is not required. Secondary leakage is addressed through SLA 1; however the leakage discount is a default value of $20 \%$, regardless of project or market conditions. The CFS approach to leakage has been classified as PLA 4 due to its comprehensive accounting for identifiable and unidentifiable leakage. However, in some aspects it resembles PLA 5 as the displacement fractions used to determine leakage effects are in principle discount factors based on area measurements within the project area. The shortcomings are that leakage is not monitored while the ex-ante estimates are based on undefined sources (see description of standard above).

\subsubsection{Leakage Effects should Be Captured on at Least National Scale}

\subsubsection{Methodological Approaches}

National scale leakage accounting is ensured by all three secondary leakage approaches (with SLA3 partly also covering international scale), but only by three of the six primary leakage approaches, as PLA 2, 3 and 6 only detect local leakage effects [52]. PLA 4 is suitable to comprehensively address national leakage, while PLA 1 and 5 have some limitations. PLA 5 uses a generic leakage discount regardless of whether or where to displacement actually occurs, thus rather theoretically accounting for leakage on all geographical scales. PLA 1 follows only the main deforestation agent in all his actions throughout the country, so that leakage tracking could in theory be avoided through the formation of subsidiary companies or outsourcing of logging activities to a third party.

\subsubsection{Carbon Accounting Standards}

All carbon standards include leakage methods that more or less effectively cover the national scale, although the main focus of the CDM and CFS methods is the local scale. CAR and VCS cover the national scale in several ways, mainly through the scope of their secondary leakage methods, but also through some of the primary leakage approaches. While the VCS applies PLA4 to address activity shifting on a national scale, the CAR uses PLA 5 with the identified shortcomings (see above).

While all standards thus fulfill the geographical scale requirement, it is remarkable that all the carbon accounting standards and most of the peer-reviewed scientific methods work under the assumption that “a country's responsibility for carbon leakage stops at its border” [24] (see p. 830), thus restricting leakage accounting to the national and sub-national levels. None of the primary leakage methods and only two of the secondary leakage methods address international leakage, and both are 
scientific modeling exercises (SLA3). International leakage is thus exclusively addressed in the scientific literature, and here in only two out of eight methods assessed. While the VCS method described in SLA 2 could in principle quantify international leakage effects, it is currently exclusively used to further refine the fraction of market leakage that remains in country, rather than accounting for international displacement. This is in line with the current international emission accounting rules that refrain from assigning responsibility for international displacement processes in all emission sectors. However, the fact that a method exists within the current carbon accounting standards that includes a conceptual approach to international leakage means that, if needed, this approach could be amended to identify and quantify displacement across country borders.

Table 5 illustrates which methodological approaches and carbon accounting standards fulfill the identified REDD requirements.

Table 5. Leakage methods grouped by carbon accounting standards screened for fulfilling important leakage features in a future REDD mechanism. CDM-Clean Development Mechanism, VCS—Verified Carbon Standard, CAR—Climate Action Reserve, CFS-Carbon Fix Standard.

\begin{tabular}{|c|c|c|c|c|c|}
\hline REDD method features & Methodological approaches & CDM & VCS & CAR & CFS \\
\hline $\begin{array}{l}\text { Leakage type } \\
\text { Addressing all relevant leakage types }\end{array}$ & & & $\mathrm{x}$ & $\mathrm{x}$ & \\
\hline $\begin{array}{l}\text { Quantification tool } \\
\text { Range of appropriate assessment } \\
\text { methods }\end{array}$ & $\begin{array}{l}\text { PLA 1,2,3,4,6 } \\
\text { SLA 1,2,3 }\end{array}$ & $x$ & $x$ & & \\
\hline $\begin{array}{l}\text { Leakage scale } \\
\text { Accounting of leakage effects on } \\
\text { national scale }\end{array}$ & $\begin{array}{l}\text { PLA } 1,4,5 \\
\text { SLA } 1,2,3\end{array}$ & $\mathrm{x}$ & $\mathrm{x}$ & $\mathrm{x}$ & $\mathrm{x}$ \\
\hline
\end{tabular}

It appears that at present the VCS is best equipped to meet all three recommended features of leakage accounting in a future REDD mechanism. The main reason is that VCS leakage methods are the only ones that combine primary and secondary leakage assessments if needed, while all other methods assessed exclusively focus on either one type of leakage (e.g., CAR, scientific methods) or are restricted to primary leakage (e.g., CFS and CDM). A national leakage accounting scale is in principle reached by all standards; although to varying degrees of accuracy (see above). CDM and VCS include the most appropriate quantification tools, so that overall, the VCS comes out as the only standard that addresses all three requirements.

When considering existing methodological approaches regardless of carbon accounting standards, a combination of suitable approaches could allow meeting the identified requirements in a comprehensive way. All three approaches to secondary leakage (SLA1-3) cover the national scale and apply appropriate tools. To also meet the leakage type requirement, any of these approaches could be combined with an approach to primary leakage that also covers the national scale and employs appropriate tools, i.e., PLA 1 or 4. Of these, PLA 4, which distinguishes geographically constrained and unconstrained leakage effects, is considered the most comprehensive approach to leakage. PLA 1 can only be used if the deforestation agent is known, and it carries the risk of leakage effects to be missed if the deforestation 
agent outsources logging activities or the forms a subsidiary logging company that is not monitored under the methodology.

\section{Conclusions}

In conclusion, we find that a number of existing methodological approaches to leakage quantification are suitable to address leakage in a future REDD mechanism, as long as it happens within national borders. Based on the 34 assessed leakage quantification methods, nine main methodological categories have been identified that apply common approaches to leakage. Six of these approaches address primary leakage from activity shifting; three address secondary leakage from market effects. All of the latter (SLA 1-3) and three of the former (PLA 1,4,5) account for leakage on the national scale as required in a REDD mechanism that follows current international emission accounting rules. All secondary leakage approaches (SLA 1-3) and five primary leakage approaches (PLA 1,2,3,4,6) use appropriate tools for leakage quantification. Thus, selected primary and secondary leakage approaches could be combined in order to meet the requirements for comprehensive leakage accounting in a future REDD mechanism. This means that a combination of currently available methods allows for comprehensive accounting of national-scale leakage emissions from REDD projects in the current voluntary and compliance carbon markets. In a future national-scale REDD mechanism these methods could be applied alongside national inventories to identify and mitigate leakage drivers to enhance effectiveness and efficiency of REDD activities.

In terms of carbon accounting standards, the VCS has been found to be best suitable at present to fulfill basic leakage accounting requirements in REDD. One reason for this could be that the VCS methodologies for REDD and IFM projects have been developed with the potential establishment of a global REDD mechanism in mind. Several methodologies exist that combine appropriate primary and secondary leakage approaches (VM0003,4,10,11,12). Both in terms of geographical scale and leakage types, VCS methods are more comprehensive than other carbon accounting standards, and quantification tools used are sound and appropriate. While the CAR Forest Protocol comes out second best in terms of leakage features formally addressed, the use of generic default discounts only is considered less appropriate.

An important finding is the lack of methods that address international leakage, which could be rampant in REDD and has the potential to seriously undermine its effectiveness. Only two methods have been identified that address this phenomenon; both are from peer- reviewed scientific articles and involve complex modeling exercises. While these are appropriate tools for leakage quantification, they require complex data input and are currently not used in on-the-ground forest conservation activities. In line with the international emissions accounting policy framework, all carbon standards assessed explicitly exclude accounting for international leakage. This is a way to keep accounting pragmatic but does not reflect the potentially severe climate and efficiency impacts of emissions displacement across country borders. As the identified quantification methods for international carbon leakage seem quite limited, a better insight into existing approaches to this issue could be obtained from literature on the broader topic of land use displacement processes. 


\section{Acknowledgments}

This research was financially supported by the Swedish Energy Agency and Forskning \& Framtid. We would like to thank Martin Persson and Johannes Ebeling for review and valuable feedback, as well as three anonymous reviewers for constructive comments.

\section{Conflict of Interest}

The authors declare no conflict of interest.

\section{References and Notes}

1. The process around REDD has developed to present REDD+ where the + refers to forest conservation, sustainable forest management and enhancement of forest carbon stocks. For the scope of this study we refer to REDD as any activities related to maintaining and enhancing forest to keep carbon stored in the biomass.

2. Cerbu, G.A.; Swallow, B.M.; Thompson, D.Y. Locating REDD: A global survey and analysis of REDD readiness and demonstration activities. Environ. Sci. Policy 2011, 14, 168-180.

3. Angelsen, A. The 3 REDD 'I’s. J. For. Econ. 2010, 16, 253-256.

4. Skutsch, M.; Bird, N.; Trines, E.; Dutschke, M.; Frumhoff, P.; de Jong, B.H.J.; van Laake, P.; Masera, O.; Murdiyarso, D. Clearing the way for reducing emissions from tropical deforestation. Environ. Sci. Policy 2007, 10, 322-334.

5. Palmer, C. Property rights and liability for deforestation under REDD+: Implications for 'permanence' in policy design. Ecol. Econ. 2011, 70, 571-576.

6. Intergovernmental Panel on Climate Change (IPCC). Special Report on Land Use, Land Use Change, and Forestry; Watson, R.T., Noble, I.R., Bolin, B., Ravindranath, N.H., Verardo, D.J., Dokken, D.J., Eds.; Cambridge University Press: Cambridge, UK, 2000.

7. Murray, B.C. Leakage from an Avoided Deforestation Compensation Policy: Concepts, Empirical Evidence, and Corrective Policy Options; Working Paper of Nicholas Institute for Environmental Policy Solutions; Duke University, Durham, NC, USA, 2008; NI WP 08-02.

8. Chomitz, K.M. Baseline, leakage and measurement issues: How do forestry and energy projects compare? Clim. Policy 2002, 2, 35-49.

9. Schwarze, R.; Niles, J.; Olander, J. Understanding and managing leakage in forest-basedgreenhouse-gas-mitigation projects. Philos. Trans. A 2002, 360, 1685-1703.

10. Myers Madeira, E.C. Policies to Reduce Emissions from Deforestation and Degradation (REDD) in Developing Countries; Atwater, S., Foerster, A., Eds.; Resources for the Future: Washington DC, USA, 2008.

11. UNFCCC: Methodological guidance for activities relating to reducing emissions from deforestation and forest degradation and the role of conservation, sustainable management of forests and enhancement of forest carbon stocks in developing countries. Decision 4 /CP15; UNFCCC: Bonn, Germany, 2009; Document Reference FCCC/CP/2009/11/Add.1. 
12. UNFCCC: The Cancun Agreements. Part C: Policy approaches and positive incentives on issues relating to reducing emissions from deforestation and forest degradation in developing countries; and the role of conservation, sustainable management of forests and enhancement of forest carbon stocks in developing countries. Decision 1/CP.16; UNFCCC: Bonn, Germany, 2010; Document Reference FCCC/CP/2010/7/Add.1.

13. Fisher, B.; Lewis, S.L., Burgess, N.D.; Malimbiwi, R.E.; Munishi, P.K.; Swetnam, R.D.; Turner, R.K.; Willcock, S.; Balmford, A. Implementation and opportunity costs of reducing deforestation and forest degradation in Tanzania. Nat. Clim. Change 2011, 1, 161-164.

14. Herold, M. An assessment of national forest monitoring capabilities in tropical non-Annex I countries: Recommendations for capacity building. The Prince's Rainforests Project: London, UK; the Government of Norway: Oslo, Norway, 2009.

15. Aukland, L.; Moura Costa, P.; Brown, S. A conceptual framework and its application for addressing leakage: The case of avoided deforestation. Clim. Policy 2003, 3, 123-136.

16. Ebeling, J.; Yasué, M. Generating carbon finance through avoided deforestation and its potential to create climatic, conservation and human development benefits. Philos. Trans. B 2008, 363, 1917-1924.

17. Wunder, S. How should we deal with leakage? In Moving Ahead with REDD: Issues, Options and Implications; Angelsen, A., Ed.; CIFOR: Bogor, Indonesia, 2008; pp. 65-76.

18. Gan, J.; McCarl, B.A. Measuring transnational leakage of forest conservation. Ecol. Econ. 2007, 64, 423-432.

19. Sohngen, B.; Beach, R.H.; Andrasko, K. Avoided deforestation as a greenhouse gas mitigation tool: Economic issues. J. Environ. Qual. 2008, 37, 1368-1375.

20. Sathaye, J.A.; Andrasko, K. Special issue on estimation of baselines and leakage in carbon mitigation forestry projects. Mitig. Adapt Strat Glob. Change 2007, 12, 963-970.

21. Fry, I. Reducing emissions from deforestation and forest degradation: Opportunities and pitfalls in developing a new legal regime. Rev. Eur. Community Int. Environ. Law 2008, 17, 166-182.

22. De Gorter, H.; Drabik, D. Components of carbon leakage in the fuel market due to biofuel policies. Biofuels 2011, 2, 119-121.

23. Sun, B.; Sohngen, B. Set-asides for carbon sequestration: Implications for permanence and leakage. Clim. Change 2009, 96, 409-419.

24. Sohngen, B.; Brown, S. Measuring leakage from carbon projects in open economies: A stop timber harvesting project in Bolivia as a case study. Can. J. For. Res. 2004, 34, 829-839.

25. Murray, B.C.; McCarl, B.A.; Lee, H. Estimating leakage from forest carbon sequestration programs. Land Econ. 2004, 80, 109-124.

26. Ewers, R.M; Rodrigues, A.S.L. Estimates of reserve effectiveness are confounded by leakage. Trends Ecol. Evol. 2008, 23, 113-116.

27. Dutschke, M.; Butzengeiger, S.; Michaelowa, A. A spatial approach to baseline and leakage in CDM forest carbon sinks projects. Clim. Policy 2006, 5, 517-530.

28. Boer, R.; Wasrin, U.R.; Perdinan, H.; Dasanto, B.D.; Makundi, W.; Hero, J.; Ridwan, M.; Masripatin, N. Assessment of carbon leakage in multiple carbon-sink projects: A case study in Jambi province, Indonesia. Mitig. Adapt Strat Glob. Change 2007, 12, 1169-1188. 
29. Lasco, R.D.; Pulhin, F.B.; Sales, R.F. Analysis of leakage in carbon sequestration projects in forestry: A case study of upper magat watershed, Philippines. Mitig. Adapt Strat Glob. Change 2007, 12, 1189-1211.

30. Sohngen, B.; Mendelsohn, R.; Sedjo, R. Forest management, conservation, and global timber markets. Am. J. Agric. Econ. 1999, 81, 1-13.

31. Sohngen, B.; Sedjo, R. Potential carbon flux from timber harvests and management in the context of a global timber market. Clim. Change 2000, 44, 151-172.

32. Wear, D.N.; Murray, B.C. Federal timber restrictions, interregional spillovers, and the impact on US softwood markets. J. Environ. Econ. Manag. 2004, 47, 307-330.

33. Zhang, J.; Gan, J. Who will meet China's import demand for forest products? World Dev. 2007, 35, 2150-2160.

34. Meyfroidt, P.; Lambin, E.F. Forest transition in Vietnam and displacement of deforestation abroad. Proc. Natl. Acad. Sci. USA 2009, 106, 16139-16144.

35. Meyfroidt, P.; Rudel, T.K.; Lambin, E.F. Forest transitions, trade, and the global displacement of land use. Proc. Natl. Acad. Sci. USA 2010, 107, 20917-20922.

36. Ristea, C.; Maness, T. Opportunities, challenges and markets for forest carbon offset projects. For. Chron. 2009, 85, 715-718.

37. Merger, E.; Dutschke, M.; Verchot, L. Options for REDD+ voluntary certification to ensure net GHG benefits, poverty alleviation, sustainable management of forests and biodiversity conservation. Forests 2011, 2, 550-577.

38. Hamilton, K.; Chokkalingam, U.; Bendana, M. State of the Forest Carbon Market 2009. Taking Root and Branching Out; Market Report by Ecosystem Marketplace; Ecosystem Marketplace: Washington, DC, USA, 2010. Available online: http://www.ecosystemmarketplace.com (accessed on 01 July 2011).

39. Kossoy, A.; Ambrosi, P. States and Trends of the Carbon Market 2010; Carbon Finance at The World Bank: Washington, DC, USA, 2011.

40. Clean Development Mechanism (CDM): Approved Large-Sale and Small-Scale Afforestation and Reforestation Methodologies; UNFCCC: Bonn, Germany, June 2011. Available online: http://cdm.unfccc.int/methodologies/index.html (accessed on 01 June 2011).

41. Forest Project Protocol Version 3.2; Climate Action Reserve: Los Angeles, CA, USA, June 2011. Available online: http:/www.climateactionreserve.org/how/protocols/forest/current/ (accessed on 18 June 2011).

42. The Verified Carbon Standard (VCS). Agriculture, Forestry and Land Use (AFOLU) Methodologies and Modules; VCS: Washington, DC, USA, June 2011. Available online: http://www.v-c-s.org/methodologies/find-a-methodology?title=\&tid=14 (accessed on 15 June 2011).

43. The CarbonFix Standard Version 3.1; Carbon Fix Standard: Stuttgart, Germany, June 2011. Available online: http://www.carbonfix.info/CarbonFix-Standard.html (accessed on 18 June 2011).

44. Skutsch, M.M.; McCall, M.K. Reassessing REDD: Governance, markets and the hype cycle. An editorial comment. Clim. Change 2010, 100, 395-402.

45. Angelsen, A. Moving Ahead with REDD: Issues, Options and Implications; Center for International Forestry Research (CIFOR): Bogor, Indonesia, 2008. 
46. Persson, U.M.; Azar, C. Preserving the world's tropical forests: A price on carbon may not do. Environ. Sci. Technol. 2010, 44, 210-215.

47. The Verified Carbon Standard (VCS). Agriculture, Forestry and Other Land Use (AFOLU) Requirements; VCS: Washington, DC, USA, 19 October 2011. Available online: http://www.v-cs.org/sites/v-c-s.org/files/AFOLU\%20Requirements\%2C\%20v3.1_1.pdf (accessed on 15 June 2011).

48. PRA is one of the tools used to gather information on community resources and needs, often applied in community development programs. It includes use of transect walks, maps, calendars, and diagrams using locally available materials.

49. Executive Board of the Clean Development Mechanism (CDM): Definition of Renewable Biomass; UNFCCC: Bonn, Germany, 2006. Available online: http://cdm.unfccc.int/EB/ Meetings/023/eb23_repan18.pdf (accessed on 15 September 2011).

50. Also by the CFS standard; which however has been classified as using PLA 4 due to its accounting for identifiable and unidentifiable leakage.

51. VM0004 and VMD0011 extent the leakage factor approach by an additional logging damage factor that accounts not only for the biomass harvested, but for the non-commercial part of the felled tree (branches and stump) and neighboring trees that are incidentally destructed during harvest; thus reflecting the emissions eventually caused per unit of extracted timber.

52. This is true for the three modeling approaches to primary leakage described under PLA 6; however the national scale requirement could be fulfilled if models with a larger geographical scale were developed.

(C) 2012 by the authors; licensee MDPI, Basel, Switzerland. This article is an open access article distributed under the terms and conditions of the Creative Commons Attribution license (http://creativecommons.org/licenses/by/3.0/). 